\title{
Weight-for-age z-score as a proxy marker for diarrhoea in epidemiological studies
}

\author{
Wolf-Peter Schmidt, ${ }^{1}$ Sophie Boisson, ${ }^{1}$ Bernd Genser, ${ }^{2}$ Mauricio L Barreto, ${ }^{2}$ \\ Kathy Baisley, ${ }^{1}$ Suzanne Filteau, ${ }^{1}$ Sandy Cairncross ${ }^{1}$
}

1 Department for Infectious and Tropical Diseases, London School of Hygiene and Tropical Medicine, UK

${ }^{2}$ Instituto de Saúde Coletiva, Federal University of Bahia, Salvador, Brazil

\section{Correspondence to} Wolf-Peter Schmidt Department of Infectious and Tropical Diseases, London School of Hygiene and Tropical Medicine, Keppel Street, WC1E 7HT London, UK; Wolf-Peter Schmidt@Ishtm.ac.uk

Accepted 30 October 2009

Published Online First

1 December 2009

\section{ABSTRACT}

Background The validity of unblinded randomised trials testing interventions against diarrhoea is severely compromised by the potential for bias. Objective proxy markers for diarrhoea not relying on self-report are needed to assess the effect of interventions that cannot be blinded. Short-term changes in weight-for-age z-score (WAZ) may (due to catch-up growth) not be a clinically important marker for nutritional status. However, even a transient decrease in WAZ could indicate recent diarrhoea, and be interpreted as the effect of an intervention.

Methods Using data from two large vitamin A trials from Ghana and Brazil, the immediate effect of the cumulative diarrhoea occurrence over 14 and 28 day time windows on WAZ was explored.

Results A very strong linear association was found between the number of days with diarrhoea over the last 14-28 days and WAZ. In both trials, differences in diarrhoea between the trial arms were associated with corresponding differences in WAZ.

Conclusion Repeated WAZ measures appear to be a suitable proxy marker for diarrhoea in children, but have disadvantages in terms of specificity and study power.

\section{INTRODUCTION}

Diarrhoeal diseases continue to be a major cause of mortality and morbidity in children. Control measures have had some success in recent years. Treatment of diarrhoea with oral rehydration is effective in reducing case fatality. ${ }^{1}$ Prevention has proved more difficult. It is hoped that in the future rotavirus vaccination may contribute to reducing diarrhoea-related mortality in poor settings. ${ }^{2}$ However, it is possible that diarrhoea will remain a leading cause of child mortality worldwide also in the future. Some of the most challenging global issues, such as nutrition, environmental pollution, population increase and climate change are not only closely related to each other, but also have the potential to contribute to living conditions associated with a high risk of diarrhoea. ${ }^{3}$ There is clearly an ongoing need for large-scale epidemiological studies to test new interventions against diarrhoea.

In the past, trials testing, for example, water, sanitation and hygiene interventions have demonstrated large reductions in diarrhoea incidence and prevalence, often ranging from $30 \%$ to $50 \%$ disease reduction (figure 1). However, the comparison of blinded and unblinded randomised trials testing the effect of household water treatment (one of the few environmental interventions against diarrhoea that can be blinded) suggests that observer and responder bias in the collection of self-reported diarrhoea data may be substantial. ${ }^{7}$ Even an observed reduction in reported diarrhoea of $50 \%$ may be compatible with no true effect (figure 1). ${ }^{7}$ A study on a portable filter device conducted in Ethiopia found a 25\% reduction of reported diarrhoea despite very low and inconsistent use, a result that is best explained by bias. ${ }^{8}$ It must be assumed that results from trials testing other unblinded interventions against diarrhoea, such as hand washing or sanitation, include a similar degree of bias. Empirical studies in other research fields, for example, clinical epidemiology, also found strong evidence for substantial bias due to lack of blinding.

Public health policy decisions aiming at reducing the burden of diarrhoea are severely compromised by this lack of reliable data on the effect of interventions, which may lead to favouring the implementation of clinical interventions that can be tested in double-blind randomised trials. ${ }^{3}$ Biased effect estimates may also cause interventions against diarrhoea to be scaled up prematurely, perhaps drawing attention away from other, possibly more beneficial public health measures. ${ }^{7}$ Alternative measures of diarrhoea that do not depend on self-report are needed to improve confidence in studies measuring the effect of unblinded diarrhoea trials. Diarrhoea-specific or overall mortality is a good outcome measure from the public health perspective, but mortality studies require a large sample size, which is beyond the scope of most trials. Health care utilisation (preferably self-initiated, not referred to by a field worker) has been used as an outcome measure in the past, ${ }^{10}$ but bias in healthcare seeking behaviour cannot be fully excluded. Other potential proxy markers for diarrhoea include inflammatory markers, ${ }^{11}$ and antibody titres against specific organisms, but results so far have been inconclusive. Also, conducting laboratory tests in large studies is logistically challenging and cost-intensive

Finally, the effect of an intervention on diarrhoea may be estimated from its effect on nutritional status. The effect of diarrhoea on weight and height is well documented in the literature. ${ }^{12-16}$ The long-term effect of diarrhoea on height and weight may be particularly pronounced in children under 6 months, ${ }^{14} 1617$ and appears to be modified by many factors such as breast feeding. ${ }^{16}$ Weight may be an inadequate marker for nutritional status, because the effect of diarrhoea on weight is thought to be rather short term and perhaps of little clinical importance. However, if weight, measured, for example, as weight-for-age z-scores (WAZ), is used only as a proxy marker for diarrhoea 
Figure 1 All an illusion? Combined estimates of the effect of environmental health interventions on diarrhoea. The first four estimates are from systematic reviews including random effects metaanalysis. ${ }^{4-6}$ The estimate of the effect of blinded household water treatment interventions is a simple average from the few published and unpublished studies, $^{7}$ as no valid data on the SEs were available. The effect size is given for illustration only - none of the blinded studies showed any evidence for an effect of the intervention on diarrhoea. ${ }^{7}$

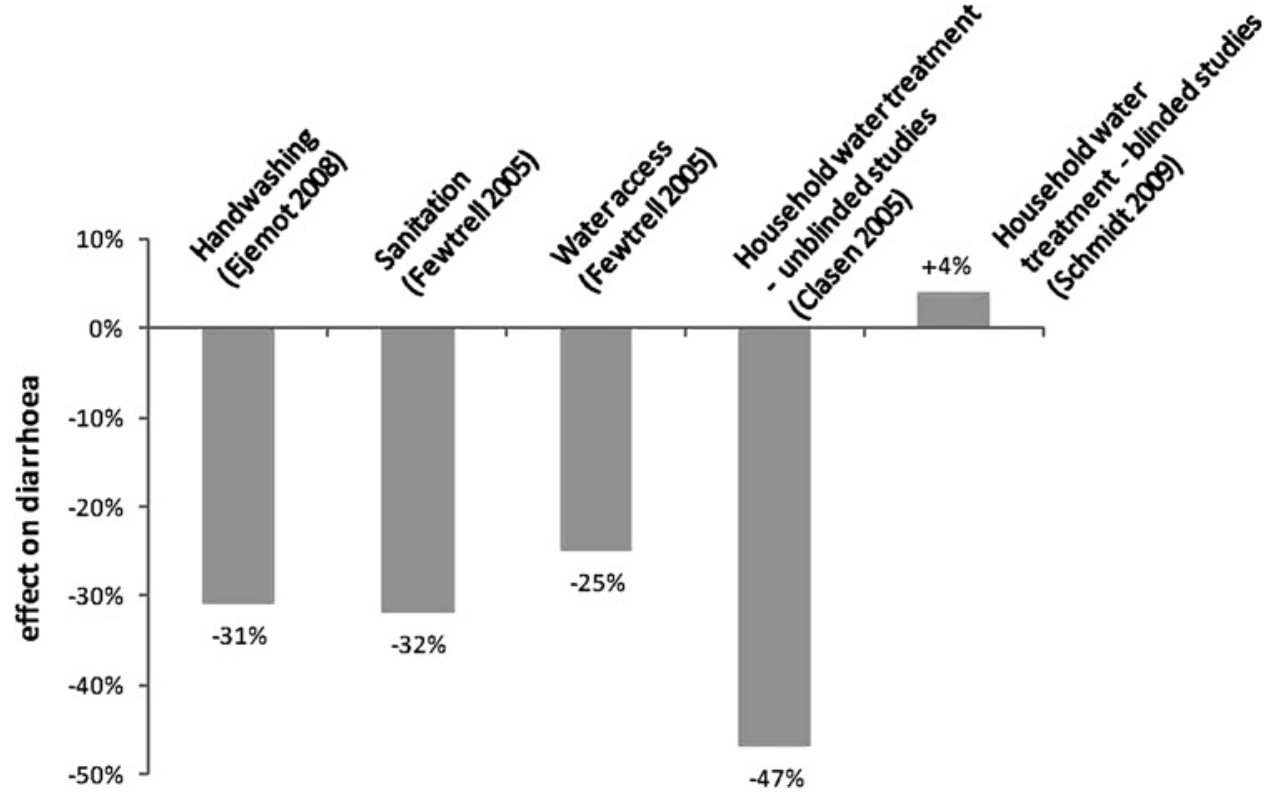

such concerns are irrelevant. It does not matter whether the observed weight loss is clinically important or largely due to transient effects of diarrhoea such as dehydration, loss of appetite, or an empty digestive system, and quickly reversed by catch-up weight gain. Although most studies on the effect of diarrhoea on nutritional status have tried to estimate the longer term effect of diarrhoea on weight, this analysis focuses on the immediate effect of diarrhoea on weight. The aim is to explore whether WAZ (which can be measured quite easily by field workers with basic training) can be used as a proxy marker of diarrhoea

\section{METHODS}

\section{Study populations}

Data from two large trials testing the effect of vitamin A supplementation on diarrhoeal and other illnesses were used. The trials were conducted in northern Ghana ${ }^{18}$ and north-eastern Brazil, ${ }^{19}$ both in semi-arid low-income settings, between 1990 and 1991. The Ghana study took place in a poor rural setting with extended families living in compounds and practicing subsistence farming. The study area was chosen due to a high prevalence of vitamin A deficiency. All compounds in the study area were included in the trial. All children aged 0-60 months living in these compounds were eligible for inclusion. The study was a dynamic cohort including newborn children and excluding children growing older than 60 months. The Brazil trial was conducted in Serrinha, a town with 30000 inhabitants $170 \mathrm{~km}$ north-west of Salvador, the capital of Bahia state. At the beginning of the study, children were identified by a census of the poorest neighbourhoods in the area. Eligible children were between 6 and 48 months of age, and had no active xerophthalmia, recent measles or recent vitamin A supplementation. In both trials children were followed up for approximately 1 year, with approximately $10 \%$ of person time lost to follow-up (table 1). Socioeconomic status was poorer in Ghana, where a large majority of households relied on open wells, bore holes and rivers as water sources. In Brazil, over $95 \%$ of households had piped-in water supply and over $90 \%$ had electricity.

\section{Diarrhoea measurement}

Both studies aimed at obtaining continuous daily records of diarrhoea, from which diarrhoea prevalence over the last 2 weeks could be obtained for the purposes of this analysis. In Ghana, weekly field worker visits measured the daily prevalence of diarrhoeal disease symptoms since the last visit; in Brazil field workers visited up to three times a week. In the Ghana dataset, diarrhoea was defined based on mothers' definitions and local disease perception. In the Brazil data, diarrhoea was defined as the occurrence of three or more loose stools over $24 \mathrm{~h}$.

It has been recognised that the adverse effects of diarrhoea such as malnutrition depend not so much on the number of diarrhoea episodes, but on the duration of these episodes ${ }^{20}$ as well as the number of days with diarrhoea over a certain period of time, also known as the longitudinal prevalence. ${ }^{15} 1621$ Therefore, a measure of recent diarrhoea was the number of days with diarrhoea in an individual during a fixed time window looking back from the date on which weight was measured. In order to focus on the short-term effect of diarrhoea on WAZ, 14day window was used for the primary analysis. In a secondary

Table 1 Epidemiological characteristics of the two study populations

\begin{tabular}{lll}
\hline & Ghana & Brazil \\
\hline Number of children & 1877 & 1209 \\
Person years & 1455 & 1104 \\
Age range at baseline (months) & $0-59$ & $6-48$ \\
Diarrhoea & & \\
$\quad$ Incidence rate/PY & 9.0 & 7.0 \\
$\quad$ Longitudinal prevalence & $17 \%$ & $5 \%$ \\
$\quad$ Mean duration of episodes (days) & 6.1 & 2.7 \\
Mean WAZ by age at measurement & & \\
$0-12$ months & -1.56 & -0.51 \\
$>12-24$ months & -1.86 & -0.89 \\
$>24-36$ months & -1.70 & -0.81 \\
$>36-48$ months & -1.57 & -0.84 \\
$>48-60$ months & -1.63 & -0.89 \\
$0-60$ months & -1.67 & -0.83 \\
WAZ $<-2$ & $30 \%$ & $13 \%$ \\
Mean HAZ & -2.39 & -1.30 \\
HAZ $<-2$ & $62 \%$ & $25 \%$ \\
Mortality & & \\
Deaths (n) & 77 & 4 \\
Mortality rate/1000 PY & 52.9 & 3.6 \\
\hline
\end{tabular}

$\mathrm{PY}$, person years of observation; WAZ/HAZ, weight/height-for age z-score. 
Figure 2 Association between diarrhoea in the last 14 days and weight-for-age z-score (WAZ) in two vitamin A trials in Ghana and Brazil. Diarrhoea was treated as categorical variable with 0 (zero) days of diarrhoea in the last 14 days as reference.

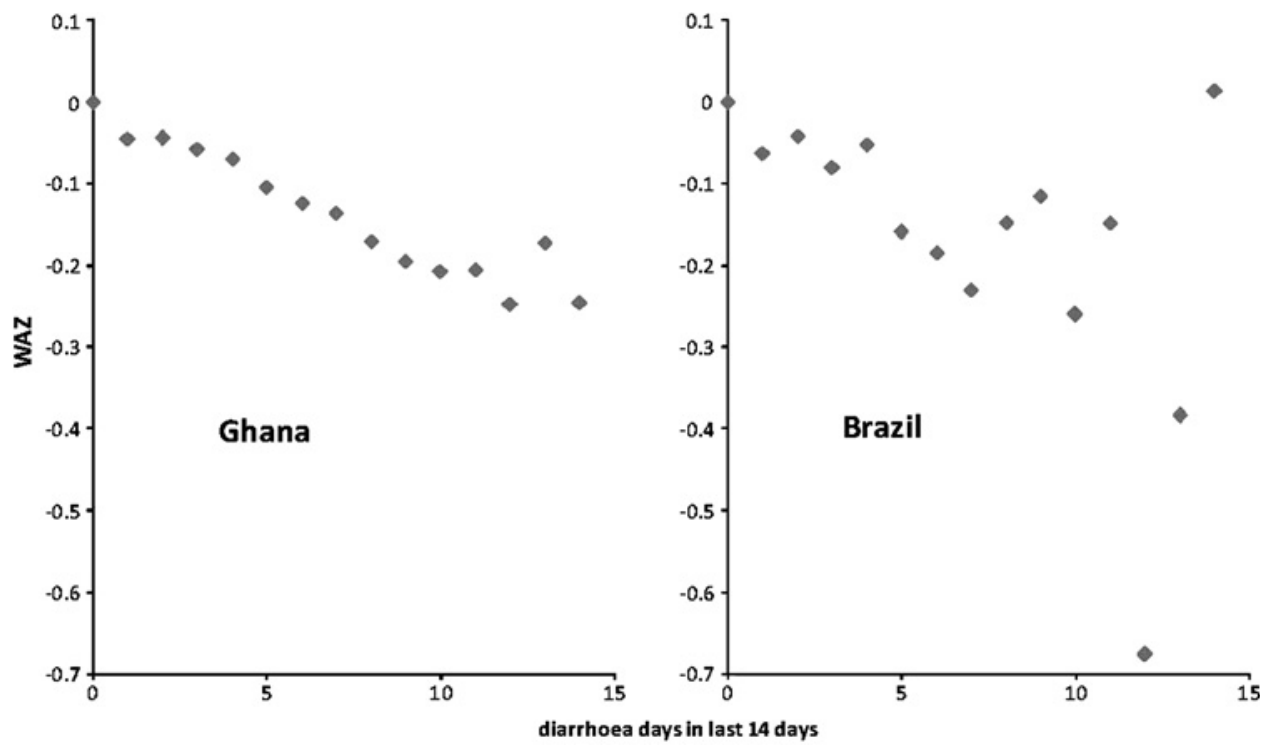

analysis, 28-day windows were also tested. Weight measurements preceded by fewer than 14 days (or 28 days) of observation were excluded from the analysis.

\section{Weight measurement}

Weight and height were measured monthly for 1 year in the Ghana study, and four times in the Brazil study (at baseline, and then every 4 months). The first measurement in the Brazil data was excluded as no diarrhoea data were available prior to the measurement.

In both trials, measurements of length/height and weight were taken in duplicate by two independent trained field workers who were blinded to the intervention. The final measure was the mean of these two measurements. Weight was measured by using hanging scales in Ghana and by electronic scales in Brazil. Weight and height were transformed into zscores (WAZ and HAZ), indicating how many standard deviations an individual child of a certain age deviates from the norm. The WHO growth norms were used for the Ghana data (using the WHO Anthro software http://www.who.int/childgrowth/ software/en/) and the national growth standard for Brazil (calculated previously ${ }^{22}$ ). HAZ was included in the analysis as a control for the analysis. It was expected than an effect of diarrhoea would be seen over the last 2 weeks on WAZ but not on HAZ

\section{Statistical analysis}

Linear regression analysis was used with WAZ (or HAZ) as the outcome variable and the number of diarrhoea days over the 14 days preceding the day of the weight or height measurement as the exposure variable. The analysis was adjusted for repeated measurement in a single child using fixed effects linear regression. Specifying a fixed effects model allowed exploring exclusively the within-child effect of diarrhoea on WAZ/HAZ, excluding potential confounding due to children with high risk of diarrhoea also having lower WAZ/HAZ scores unrelated to diarrhoea. In both Ghana and Brazil, older children had on average lower WAZ than children under the age of 12 months. At the same time, older children had less diarrhoea than younger children. Therefore, age needed to be included as a confounding variable. Age was included in the model by constructing a restricted cubic spline (three knots), as the association between age and diarrhoea was non-linear.
The effect of the intervention (vitamin A) on WAZ was analysed using random effects linear regression adjusted for repeated measurements in a child. The analysis was adjusted for the baseline WAZ measurement prior to the intervention. The effect of the intervention on the number of diarrhoea days over the 14 days prior to anthropometric measurements was analysed using random effects Poisson regression. The analysis was done in STATA 10 (StataCorp, Texas, USA).

\section{RESULTS}

Epidemiological and growth status characteristics of the two study populations are shown in table 1 . The children in Ghana had a higher risk of diarrhoea, underweight (WAZ $<-2)$, growth faltering $(\mathrm{HAZ}<-2)$ and death. The mean duration of diarrhoea episodes was much longer in Ghana than in Brazil.

In a first step, the number of diarrhoea days in the past 14 days was treated as a continuous variable. All analyses were adjusted for age at the time of measurement (restricted cubic spline), which did not influence the slope but narrowed the confidence intervals. In Ghana, WAZ declined by 0.018 with every additional day with diarrhoea (95\% CI -0.020 to -0.016$)$, in Brazil by 0.022 (95\% CI -0.028 to -0.015$)$. In a post hoc analysis, a trend was found suggesting that the association between diarrhoea and WAZ was slightly stronger in children under 24 months compared to over 24 months (choosing 12 months of age as cut-off point showed similar results). In Ghana, the regression coefficients for children under or above 24 months were -0.019 and -0.013 respectively (test for interaction $\mathrm{p}=0.07)$. In Brazil, the regression coefficients were 0.029 and 0.021 respectively (test for interaction $p=0.08$ ).

Figure 2 shows the results of the regression analysis with the number of diarrhoea days in the past 14 days treated as a categorical variable, with no (zero) diarrhoea days as reference. In both study populations there is a very pronounced linear association between the number of days with diarrhoea and WAZ.

To test the appropriateness of the regression model, HAZ was used instead of WAZ as the outcome variable. As expected, there was no evidence for an association between the number of days with diarrhoea over the last 14 days and HAZ in Ghana (coefficient $-0.003,95 \% \mathrm{CI}-0.007$ to 0.002). In Brazil, diarrhoea was associated with a slight increase in HAZ (coefficient 0.007, 95\% CI 0.001 to 0.013 ). Figure 3 shows the same analysis with the 
Figure 3 Association between diarrhoea in the last 14 days and heightfor-age z-score (HAZ) in two vitamin A trials in Ghana and Brazil. Diarrhoea was treated as categorical variable with 0 (zero) days of diarrhoea in the last 14 days as reference.

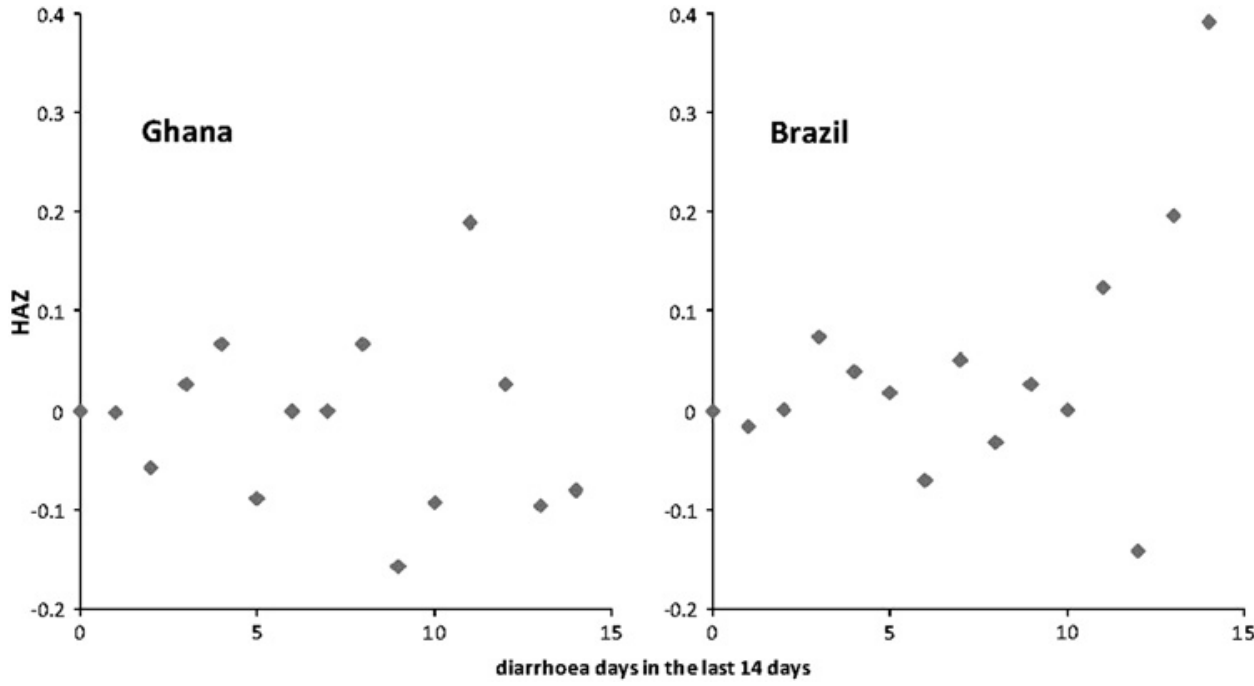

number of diarrhoea days as categorical variable (as in figure 2), confirming the lack of a negative immediate effect of diarrhoea on HAZ in both study populations.

The association between recent diarrhoea and WAZ was also apparent when using a 28 day window instead of a 14 day window for recent diarrhoea. In Ghana, WAZ declined by 0.013 standard deviations with every additional day with diarrhoea (95\% CI -0.014 to -0.012 ), in Brazil by 0.014 SDs (95\% CI -0.018 to -0.010$)$. Figure 4 shows the results of the regression analysis with the number of diarrhoea days in the past 28 days treated as a categorical variable, again with no (zero) diarrhoea days as reference, confirming the linear association between recent diarrhoea and WAZ.

Finally, it was tested whether the effect of the intervention (vitamin A) on diarrhoea and WAZ were correlated. In Ghana, vitamin A had no effect on diarrhoea prevalence. The overall prevalence ratio (PR) was 1.01 (95\% CI 0.89 to 1.14). However, if follow-up time was restricted to the 14 days prior to an anthropometric measurement, there was slight decrease in diarrhoea in the intervention arm $(\mathrm{PR}=0.97$, table 2$)$. The above analyses suggested that a reduction in diarrhoea should translate into an increase in WAZ. Accordingly, there was a slight increase in WAZ by 0.04 in the intervention arm (table 2).

In the Brazil study, vitamin A was overall associated with a $10 \%$ decline in diarrhoea longitudinal prevalence (proportion of time ill) ( $\mathrm{PR}=0.90,95 \% \mathrm{CI} 0.78$ to 1.03$)$. However, if the followup time is restricted to the 14 days prior to an anthropometric measurement, the treatment arm experienced slightly higher longitudinal prevalence $(\mathrm{PR}=1.03$, table 2$)$. Again, there were corresponding changes in the WAZ with a slight decrease of 0.03 .

(Note that the overall results for Ghana and Brazil slightly deviate from the published data, since a slightly different common analysis method was used for both datasets.)

\section{DISCUSSION}

The results demonstrate a strong linear relationship between diarrhoea in the last 2-4 weeks and weight for age z-score in children under the age of 5 , suggesting that WAZ may be a suitable proxy marker to measure the effect of an unblinded intervention against diarrhoea. Many previous diarrhoea trials, especially those testing the effect of environmental health
Figure 4 Association between diarrhoea in the last 28 days and weight-for-age z-score (WAZ) in two vitamin A trials in Ghana and Brazil. Diarrhoea was treated as categorical variable with 0 (zero) days of diarrhoea in the last 28 days as reference.

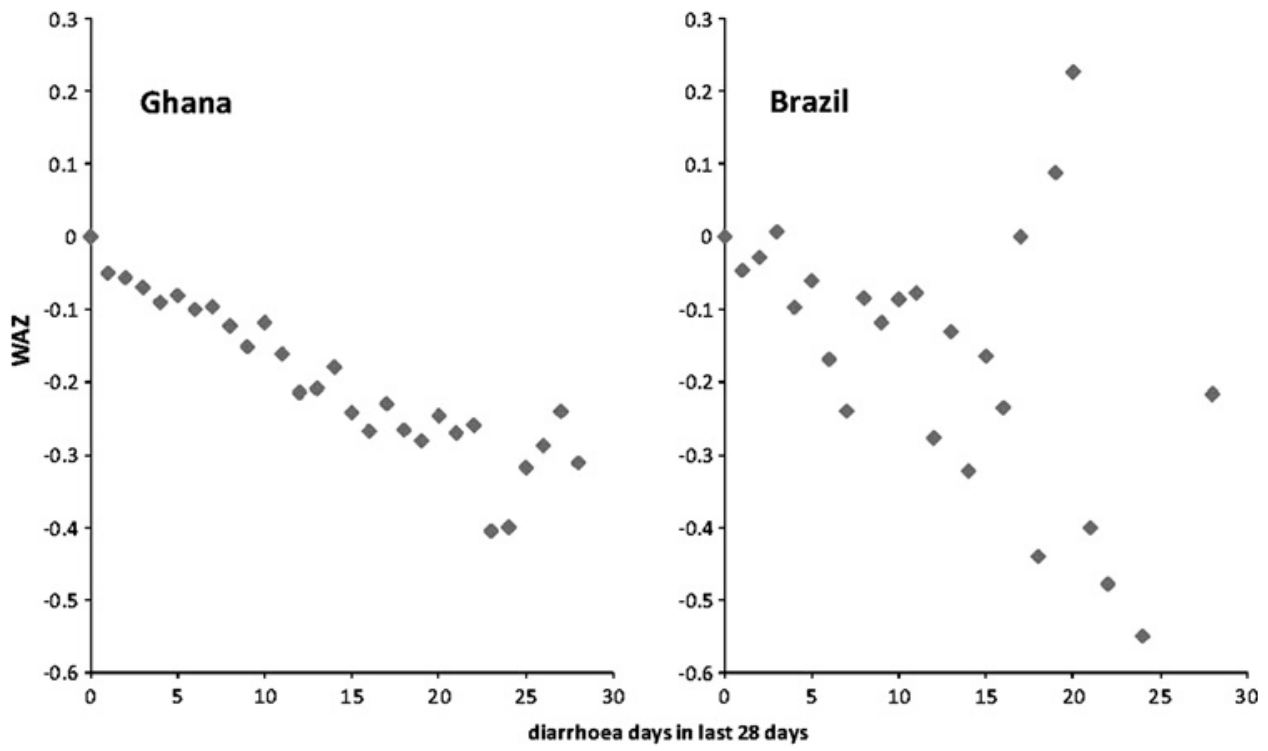


Table 2 Effect of the intervention (vitamin A) on diarrhoea in the 14 days before measurement of weight, and WAZ adjusted for baseline WAZ

\begin{tabular}{|c|c|c|c|c|}
\hline Study & $\begin{array}{l}\text { Diarrhoea } \\
\text { LP }\end{array}$ & $\begin{array}{l}\text { Diarrhoea } \\
\text { PR } \\
\text { (95\% Cl) }\end{array}$ & WAZ & $\begin{array}{l}\text { WAZ } \\
\text { difference } \\
(95 \% \mathrm{CI})\end{array}$ \\
\hline \multicolumn{5}{|l|}{ Ghana } \\
\hline Control & $17.7 \%$ & & -1.71 & \\
\hline Vitamin A & $17.3 \%$ & $\begin{array}{l}0.97 \\
(0.86 \text { to } 1.10)\end{array}$ & -1.67 & $\begin{array}{l}+0.04 \\
(-0.01 \text { to } 0.09)\end{array}$ \\
\hline \multicolumn{5}{|l|}{ Brazil } \\
\hline Control & $4.9 \%$ & & -0.82 & \\
\hline Vitamin A & $5.1 \%$ & $\begin{array}{l}1.03 \\
(0.82 \text { to } 1.29)\end{array}$ & -0.86 & $\begin{array}{l}-0.03 \\
(-0.06 \text { to } 0.00)\end{array}$ \\
\hline
\end{tabular}

LP, longitudinal prevalence (=proportion of time ill); PR, (longitudinal) prevalence ratio.

interventions on diarrhoea, have either not included WAZ as an outcome measure at all, or have measured weight only at the beginning and end of the intervention period $\left(\mathrm{eg},{ }^{10}{ }^{23}\right)$. According to this analysis, observing a strong reduction in selfreported diarrhoea, as is often the case in unblinded diarrhoea trials (figure 1), but failing to find larger mean WAZ compared to the control arm may speak against a true effect of an intervention on diarrhoea.

The major drawback of WAZs may be their low specificity. Many conditions, for example, fever or respiratory infections, can acutely alter appetite, hydration, metabolism and weight. Using WAZ as a proxy for diarrhoea is, therefore, most appropriate in trial testing an intervention that specifically reduces diarrhoea. For many water, sanitation and hygiene interventions (which appear to be particularly affected by bias) this should be the case. Any differences in WAZ between intervention and control group are most likely due to differences in diarrhoea, but at times may to some extent also be due to a decrease in helminth infections. Other interventions, for example, vitamin A or other micronutrient supplementation (which can often be blinded) may affect illnesses other than diarrhoea and may, therefore, cause changes in WAZ in the absence of an effect on diarrhoea.

The presumably low specificity of WAZ as a proxy marker for diarrhoea is likely to affect study power. Non-differential misclassification in an outcome variable does not lead to bias but lowers the precision of effect estimates. To maximise power, WAZ needs to be measured repeatedly over the study period, for example, every 4 weeks at the same time when collecting data on self-reported diarrhoea. Study power can further be strengthened by adjusting the analysis for the baseline WAZ before the intervention, as done in this analysis. Study power will depend on many factors, such as diarrhoea prevalence, prevalence of other diseases causing acute weight loss (which may lower precision), and the proportion of diarrhoea episodes promptly treated with oral rehydration (which should limit acute weight loss).

Weight-for-age z-scores are relatively easy to measure compared, for example, to laboratory markers, and should require a smaller sample size compared to objective but rare outcomes such as mortality. Weight measurements should be taken following a strict protocol and standardised equipment to minimise measurement error. ${ }^{24}$ Such measures add to the logistics of diarrhoea trials. There is also the risk of observer bias, if the field worker taking the weight measurement cannot be blinded to the intervention. However, the risk of bias seems much smaller compared to self-reported diarrhoea.

The relationship between diarrhoea and nutritional status has been subject to intensive research. This analysis does not aim at reinventing the wheel, but simply takes a slightly different perspective by focusing on the immediate effect of diarrhoea on WAZ. The observed strong short-term effect may not be clinically important. Regained appetite and rehydration after an episode may quickly reverse weight loss. It is, therefore, important not to overinterpret any changes in WAZ with respect to the effect of an intervention on malnutrition. Applying WAZ as suggested here largely concerns the effect of an intervention on diarrhoea. Further, WAZ is unlikely to substantially contribute to the interpretation of observational studies on diarrhoea. Here, the major challenge in the analysis lies in the very large potential for socioeconomic confounding, which is difficult if not impossible to address analytically. ${ }^{25}$ WAZ may be no less affected by confounding than self-reported diarrhoea.

This analysis was restricted to children under the age of 5 years. Presumably, diarrhoea acutely affects weight also in older individuals. Often, it may be less practical to take repeated weight measures in adolescents and adults especially if the protocol requires weight measures with only limited clothing. Taking measures with clothes on should in most cases provide unbiased estimates between intervention and control group but increases the SE.

The ideal approach to confirm the validity of WAZ as a proxy marker for diarrhoea would be to include repeated WAZ measures as a secondary outcome measure in a double-blind randomised trial of an intervention that turns out to be effective in reducing reported diarrhoea. In a blinded trial, the estimate of the reduction in reported diarrhoea should be unbiased, as should be the effect of the intervention on WAZ. Perhaps, future vaccine trials such as rotavirus vaccination will provide such an opportunity.

In conclusion, repeated measures of weight-for-age z-score appear to be a suitable and relatively objective proxy marker for diarrhoea in unblinded trials, but have disadvantages in terms of specificity and study power. Wherever possible, blinding an intervention and using reported diarrhoea or mortality is still the method of choice. ${ }^{7}$ Given that results of unblinded trials on reported diarrhoea may have little or even no validity, demonstrating the presence or absence of changes in WAZ could allow improvement of the evidence base of diarrhoea trials, especially those testing complex environmental interventions.

\section{What is already known on this subject}

- Unblinded randomised trials testing interventions against diarrhoea provide highly biased effect estimates when relying on self-report.

- Proxy markers for diarrhoea are needed to reinstall confidence in diarrhoea trials and prevent inadequate health policy decisions.

\section{What this study adds}

- In this analysis of two large randomised trials it was found that repeated measures of weight-for-age z-score could be used as a proxy marker for recent diarrhoea experience, even if it may have little clinical importance as a measure for nutritional status. 
Acknowledgements We thank Saul Morris for providing data from the Ghana VAST trial, and Ben Armstrong and Antonio Gasparrini for statistical advice.

Funding This work was supported by Wellcome Trust, UK, Grant Number: WT082569AIA.

Competing interests None.

Provenance and peer review Not commissioned; externally peer reviewed.

\section{REFERENCES}

1. Jones G, Steketee RW, Black RE, et al. How many child deaths can we prevent this year? Lancet 2003;362:65-71.

2. Zaman K. Rotavirus vaccine: a promise for the future. J Health Popul Nutr 2008; $26: 385-7$

3. Schmidt WP. Setting priorities in diarrhoeal disease research: merits and pitfalls of expert opinion. J Health Popul Nutr 2009:27:313-15.

4. Clasen T, Schmidt WP, Rabie T, et al. Interventions to improve water quality for preventing diarrhoea: systematic review and meta-analysis. BMJ 2007:334:782.

5. Ejemot RI, Ehiri JE, Meremikwu MM, et al. Hand washing for preventing diarrhoea. Cochrane Database Syst Rev 2008:(1);CD004265.

6. Fewtrell L, Kaufmann RB, Kay D, et al. Water, sanitation, and hygiene interventions to reduce diarrhoea in less developed countries: a systematic review and metaanalysis. Lancet Infect Dis 2005;5:42-52.

7. Schmidt WP, Cairncross S. Household water treatment in poor populations: is there enough evidence for scaling up now? Environ Sci Technol 2009;43:986-92.

8. Boisson S, Schmidt WP, Berhanu T, et al. A randomised controlled trial in rural Ethiopia to test a portable water treatment device. Environ Sci Technol 2009;43:5934-9.

9. Wood L, Egger M, Gluud LL, et al. Empirical evidence of bias in treatment effect estimates in controlled trials with different interventions and outcomes: metaepidemiological study. BMJ 2008;336:601-5.

10. Luby SP, Agboatwalla M, Painter J, et al. Combining drinking water treatment and hand washing for diarrhoea prevention, a cluster randomised controlled trial. Trop Med Int Health 2006;11:479-89.

11. Filteau SM, Morris SS, Raynes JG, et al. Vitamin A supplementation, morbidity, and serum acute-phase proteins in young Ghanaian children. Am J Clin Nutr 1995;62:434-8.
12. Black RE, Brown $\mathrm{KH}$, Becker S. Effects of diarrhea associated with specific enteropathogens on the growth of children in rural Bangladesh. Pediatrics 1984:73:799-805.

13. Checkley W, Buckley G, Gilman RH, et al. Multi-country analysis of the effects of diarrhoea on childhood stunting. Int J Epidemiol 2008;37:816-30.

14. Condon-Paoloni D, Cravioto J, Johnston FE, et al. Morbidity and growth of infants and young children in a rural Mexican village. Am J Public Health 1977;67:651-6.

15. Morris SS, Cousens SN, Kirkwood BR, et al. Is prevalence of diarrhea a better predictor of subsequent mortality and weight gain than diarrhea incidence? Am J Epidemiol 1996; 144:582-8.

16. Rowland MG, Rowland SG, Cole TJ. Impact of infection on the growth of children from 0 to 2 years in an urban West African community. Am J Clin Nutr 1988:47:134-8.

17. Checkley W, Epstein LD, Gilman RH, et al. Effects of acute diarrhea on linear growth in Peruvian children. Am J Epidemiol 2003;157:166-75.

18. Anon. Vitamin A supplementation in northern Ghana: effects on clinic attendances, hospital admissions, and child mortality. Ghana VAST Study Team. Lancet 1993;342:7-12.

19. Barreto ML, Santos LM, Assis AM, et al. Effect of vitamin A supplementation on diarrhoea and acute lower-respiratory-tract infections in young children in Brazil. Lancet 1994; 344:228-31.

20. Baqui AH, Black RE, Sack RB, et al. Epidemiological and clinical characteristics of acute and persistent diarrhoea in rural Bangladeshi children. Acta Paediatr Suppl 1992; 381:15-21.

21. Torres AM, Peterson KE, de Souza AC, et al. Association of diarrhoea and upper respiratory infections with weight and height gains in Bangladeshi children aged 5 to 11 years. Bull World Health Organ 2000;78:1316-23.

22. Assis AM, Barreto ML, Santos LM, et al. Growth faltering in childhood related to diarrhea: a longitudinal community based study. Eur J Clin Nutr 2005:59:1317-23.

23. Luby SP, Agboatwalla M, Feikin DR, et al. Effect of handwashing on child health: a randomised controlled trial. Lancet 2005; 366:225-33.

24. Lohmann TG, Roche AF, Martorell A. Anthropometric standardization reference manual. Champaign, IL: Human Kinetics Publications, 1988.

25. Kaufman JS, Cooper RS, McGee DL. Socioeconomic status and health in blacks and whites: the problem of residual confounding and the resiliency of race. Epidemiology 1997;8:621-8. 